\title{
The Prion-Like Behavior of Assembled Tau in Transgenic Mice
}

\author{
Florence Clavaguera ${ }^{1}$, Markus Tolnay ${ }^{1}$, and Michel Goedert ${ }^{2}$ \\ ${ }^{1}$ Institute of Pathology, University Hospital, CH-4031 Basel, Switzerland \\ ${ }^{2}$ MRC Laboratory of Molecular Biology, Cambridge CB2 0QH, United Kingdom \\ Correspondence:mg@mrc-Imb.cam.ac.uk; f.clavaguera@unibas.ch
}

Tauopathies constitute neurodegenerative diseases that are characterized by the intracellular deposition of filaments made of hyperphosphorylated tau protein. The pattern of tau deposition in Alzheimer's disease follows a stereotypical progression, with the first lesions appearing in the locus coeruleus and entorhinal cortex, from where they appear to spread to the hippocampus and neocortex. Propagation of pathological tau is also characteristic of argyrophilic grain disease, where the lesions seem to spread through distinct regions of the limbic system. In chronic traumatic encephalopathy, tauopathy appears to spread from the neocortex to the brainstem. These findings implicate neuron-to-neuron propagation of tau aggregates. Isoform compositions and morphologies of tau filaments can differ between tauopathies, which is consistent with the existence of distinct tau strains. Here, we review recent findings that support prion-like mechanisms in the pathogenesis of tauopathies through the experimental use of transgenic mice.

\section{HUMAN TAUOPATHIES}

$M_{\mathrm{i}}^{\mathrm{i}}$ icrotubule-associated protein tau (MAPT) is expressed in the adult human brain as six different isoforms that result from the alternative mRNA splicing of a single gene located on chromosome 17 (Goedert et al. 1989; Spillantini and Goedert 2013). These isoforms vary from 352 to 441 amino acids in length, depending on the number of $\mathrm{N}$-terminal inserts $(0,1$, or 2$)$ and the presence (or not) of a fourth 31 amino acid repeat encoded by exon 10 . The C-terminal repeats (residues 252-376) and some adjoining sequences form the microtubule-binding domains of tau, with four-repeat isoforms ( $4 \mathrm{R}$ tau) being better at promoting microtubule assembly than isoforms with three repeats (3R tau) (Goedert and Jakes 1990). Tau is monomeric, soluble, unfolded, and phosphorylated; however, it becomes aggregated, insoluble, and hyperphosphorylated in a large number of human neurodegenerative diseases (Table 1) (Goedert et al. 2010). These tauopathies are sporadic or caused by mutations in MAPT, the tau gene (Hutton et al. 1998; Poorkaj et al. 1998; Spillantini et al. 1998; Goedert 2015). Interestingly, the compositions and shapes of the isoforms and the cell-type localizations of tau lesions differ between tauopathies (Crowther and Goedert 2000). This heterogeneity may give rise to the diversity of human tauopathies. In Alzheimer's disease (AD), chronic traumatic

Editor: Stanley B. Prusiner

Additional Perspectives on Prion Diseases available at www.perspectivesinmedicine.org

Copyright (C) 2017 Cold Spring Harbor Laboratory Press; all rights reserved; doi: 10.1101/cshperspect.a024372

Cite this article as Cold Spring Harb Perspect Med 2017;7:a024372 
F. Clavaguera et al.

Table 1. Diseases with tau inclusions

Alzheimer's disease
Amyotrophic lateral sclerosis/parkinsonism-
dementia complex
Anti-IgLON5-related tauopathy
Argyrophilic grain disease
Chronic traumatic encephalopathy
Corticobasal degeneration
Diffuse neurofibrillary tangles with calcification
Down's syndrome
Familial British dementia
Familial Danish dementia
Frontal variant of Alzheimer's disease
Frontotemporal dementia and parkinsonism linked
to chromosome 17 caused by MAPT mutations
Gerstmann-Sträussler-Scheinker disease
Guadeloupean parkinsonism
Huntington's disease
Meningio-angiomatosis
Myotonic dystrophy
Neurodegeneration with brain iron accumulation
Niemann-Pick disease, type C
Non-Guamanian motor neuron disease with
neurofibrillary tangles
Pick's disease
Postencephalitic parkinsonism
Prion protein cerebral amyloid angiopathy
Progressive subcortical gliosis
Progressive supranuclear palsy
SLC9A6-related mental retardation
Subacute sclerosing panencephalitis
Tangle-only dementia
White-matter tauopathy with globular glial
inclusions

encephalopathy (CTE), and tangle-only dementia (TD), neuronal tau lesions are composed of 3R and 4R isoforms (Goedert et al. 1992; Schmidt et al. 2001; Noda et al. 2006). They constitute neurofibrillary tangles (NFTs), with filaments located in the somatodendritic compartment, and neuropil threads (NTs), where tau is aggregated in distal processes. In neurons of patients with Pick's disease (PiD), 3R tau isoforms predominate in Pick bodies (Delacourte et al. 1996; Probst et al. 1996). Other sporadic tauopathies, such as progressive supranuclear palsy (PSP), corticobasal degeneration (CBD), and argyrophilic grain disease (AGD), are characterized by tau inclusions made of $4 \mathrm{R}$ tau in both neurons and glia (Flament et al. 1991; Ksiezak-Reding et al. 1994; Togo et al. 2002; Tolnay et al. 2002). Neuronal tau inclusions are globose-type NFTs and NTs in PSP; they are NTs, pretangles, and small NFTs in $\mathrm{CBD}$, whereas abundant argyrophilic grains and pretangle neurons are representative of AGD (Probst et al. 1988; Feany and Dickson 1995; Tolnay and Clavaguera 2004). PSP, CBD, and AGD share similar oligodendroglial tau inclusions in the shape of coiled bodies (Yamada et al. 1992; Komori et al. 1998; Botez et al. 1999). However, they are also characterized by distinct astrocytic tau lesions defined as tufted astrocytes for PSP or AGD and astrocytic plaques for CBD (Nishimura et al. 1992; Tolnay and Probst 2002; Tolnay and Clavaguera 2004). The tufted astrocytes of PSP are Gallyas-Braak silver positive, whereas those of AGD are silver negative. Cases of frontotemporal dementia and parkinsonism linked to chromosome 17 that are caused by MAPT mutations (FTDP-17T) display filamentous tau inclusions in nerve cells and/or glial cells accompanied by severe neuronal loss, astrocytic gliosis, and spongiosis. A given mutation determines the composition of the filaments, which consist of 3R, 4R, or a mixture of all six tau isoforms (Ghetti et al. 2011). In $\mathrm{AD}, \mathrm{AGD}$, and CTE, filamentous tau inclusions propagate through the brain via connected regions, reminiscent of prions. Braak and Braak defined six stages of tau-inclusion progression based on the presence of silverstained, hyperphosphorylated tau aggregates (Braak and Braak 1991; Braak et al. 2006). In the first stages, NFTs are found in the transentorhinal and entorhinal cortex (I and II), followed by hippocampal formation and the temporal cortex (III and IV). In stages III and IV, the nerve cell damage may be severe enough to cause first clinical symptoms. Eventually, NFTs invade isocortical association areas in stages $\mathrm{V}$ and VI, resulting in severe dementia. In a more recent study, Braak and Del Tredici (2011) reported the initial accumulation of hyperphosphorylated tau in neurons of the locus coeruleus (LC). This region projects to the transentorhinal cortex, suggesting anterograde axonal transport of pathological tau, followed 
by its propagation. In AGD, three stages of disease progression are observed, with the earliest tau inclusions in the ambient gyrus (I), where they extend to the anterior and posterior medial temporal lobe (II), followed by the septum, insular cortex, and anterior cingulate gyrus (III). The clinical symptoms in stage III are representative of patients with a diagnosis of dementia (Saito et al. 2004). CTE also presents with a stereotypical propagation of hyperphosphorylated tau deposition that occurs in parallel with axonal loss. Four stages have been described, with the first inclusions starting in the depths of some cortical sulci, close to perivascular spaces. From there, they spread through the cerebral cortex, diencephalon, basal ganglia, brainstem, and spinal cord (McKee et al. 2013).

\section{EXPERIMENTAL INDUCTION AND PROPAGATION OF TAU PATHOLOGY}

Over the past 7 years, experiments using mice transgenic for human tau protein have provided evidence for neuron-to-neuron propagation of tau aggregates (Clavaguera et al. 2015; Goedert 2015). In July 2009, we were the first to show the experimental induction and propagation of tau pathology in vivo (Clavaguera et al. 2009). We used ALZ17 mice transgenic for one isoform of wild-type $4 \mathrm{R}$ tau and mice transgenic for one isoform of $4 \mathrm{R}$ tau with the P301S mutation of FTDP-17T (Probst et al. 2000; Allen et al. 2002). We injected homogenates with numerous tau aggregates prepared from P301S tau brains into the hippocampus and overlying cerebral cortex of ALZ17 mice, which do not develop filamentous tauopathy (Fig. 1A). This resulted in the assembly of wild-type human tau into filaments in neurons (NFTs and NTs) and oligodendrocytes (coiled bodies), similar to the inclusions observed in human tauopathies (Fig. 1B). The induced tauopathy did not remain confined to the injection sites. Silver-positive tau lesions progressed over time to both neighboring and more distantly connected brain regions, consistent with the intercellular transfer of tau aggregates (Fig. 1C). The presence of tau was necessary for inducing filamentous pathology, because no aggregation was observed after the inoculation of P301S homogenates immunodepleted of tau.

Since 2009, our work has been confirmed and extended. In one study, what was reported to be oligomeric tau from $\mathrm{AD}$ brains was injected into wild-type mice, which showed propagation of the induced filamentous tauopathy (Lasagna-Reeves et al. 2012). Silver-positive staining was present not only in the injected hippocampus but also in neighboring areas, such as the cerebral cortex, corpus callosum, and hypothalamus. Two groups have studied the spreading of pathological tau along the entorhinal cortex/hippocampal pathway. They used mouse models that apparently only expressed human mutant P301L tau in the entorhinal cortex (de Calignon et al. 2012; Liu et al. 2012). A time-dependent increase of tau pathology was not limited to the transgene-expressing neurons of the entorhinal cortex. Several months after the appearance of the first tau inclusions in the entorhinal cortex, hippocampal neurons also developed a filamentous tau pathology. We showed that the induction and propagation of tau aggregates in ALZ17 brains was almost entirely a result of the insoluble tau fraction from P301S brain extracts (Clavaguera et al. 2009). This was supported by us and others (Clavaguera et al. 2013b; Iba et al. 2013) with the demonstration that preformed synthetic tau fibrils (tau PFFs) assembled from human mutant recombinant protein promoted the formation of NFT-like inclusions in a dose- and time-dependent manner when injected into the brains of presymptomatic P301S tau mice. The induced tau pathology propagated to brain regions connected to the injection sites. When synthetic tau fibrils were injected into the hippocampus, fibrillar tau was found in the entorhinal cortex and the contralateral (noninjected) hippocampus, whereas following injection into the striatum, filamentous tau appeared in the substantia nigra, thalamus, and corpus callosum (Iba et al. 2013). This study was recently extended with the inoculation of tau PFFs into the LC (Iba et al. 2015). Mature NFTs formed rapidly at the sites of injection and propagated to a small number of neurons in the contralateral LC. Interestingly, the num- 
F. Clavaguera et al.

A

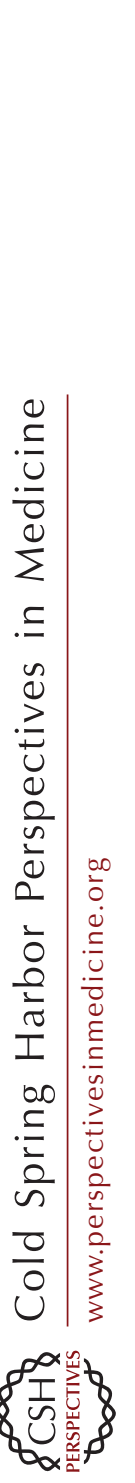

B

C
P301S transgenic mouse

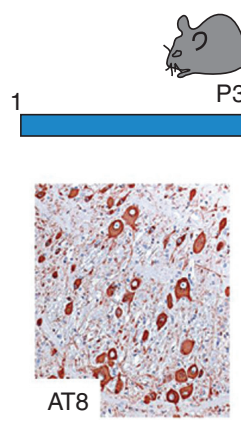

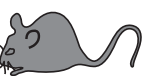

P301S mutation 383

Shortest 4R hTau

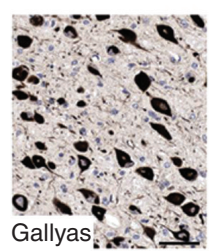

ALZ17 transgenic mouse

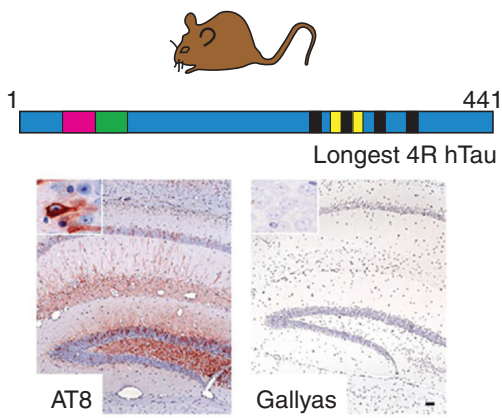

Noninjected ALZ17

P301S-injected
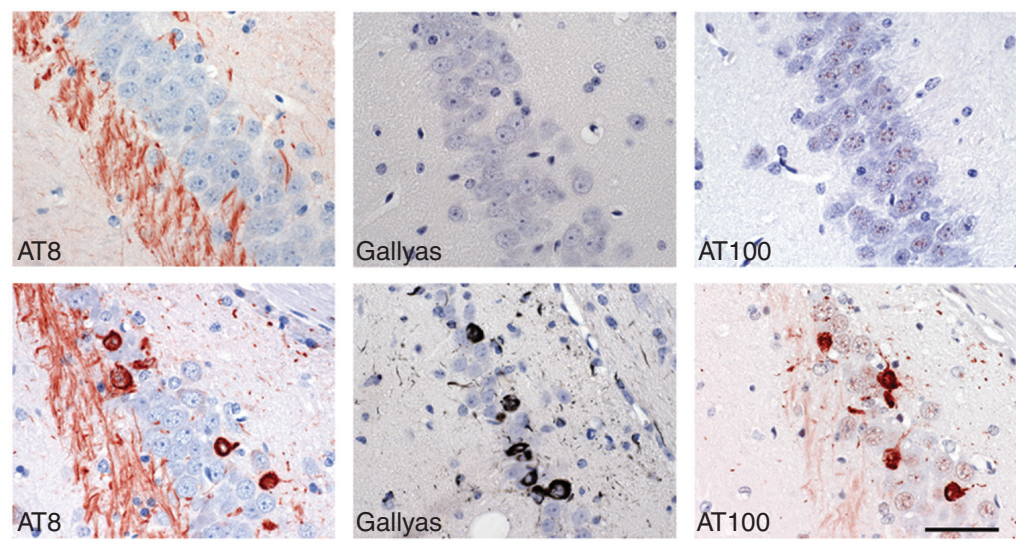

P301S-injected ALZ17
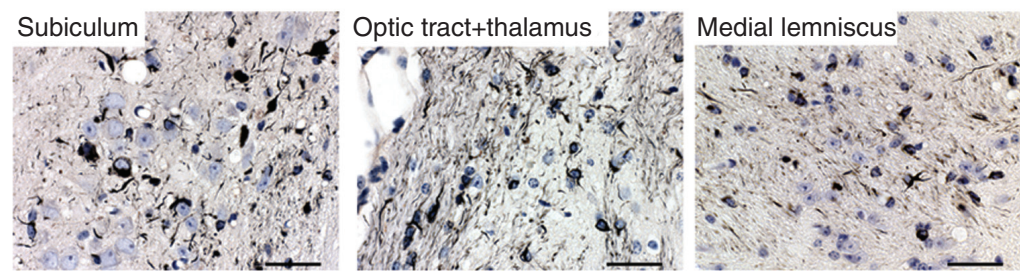

Figure 1. Induction and propagation of filamentous tauopathy in tau transgenic mouse models. (A) (Left) Mice expressing the 383 amino acid 4R human tau with the P301S mutation under the control of the murine Thy1 promoter develop abundant hyperphosphorylated (AT8-immunoreactive) and Gallyas-Braak silver-positive filamentous tau inclusions in various brain regions, including the brainstem, from which extracts were prepared for injection into the brains of ALZ17 mice. (Right) ALZ17 mice express the 441 amino acid 4R human tau isoform under the control of the murine Thyl promoter. These mice show tau that is immunoreactive with the phosphorylation-dependent antibody AT8, but Gallyas-Braak silver negative, as shown here for the hippocampus. They also lack tau filaments. The sections were counterstained with hematoxylin. $(B)$ Staining of the hippocampal CA3 region from 18-month-old ALZ17 mice with AT8, Gallyas-Braak silver and phosphorylation-dependent anti-tau antibody AT100, 15 months after no injection (upper panel) or after the injection with brain extract from 6-month-old P301S tau mice (lower panel). The injection of brain extracts induced the formation of AT100- and Gallyas-Braak silver-positive filamentous inclusions made of wild-type human tau. No such inclusions were found in ALZ17 mice injected with extracts from control mice (Clavaguera et al. 2009). The sections were counterstained with hematoxylin. $(C)$ Spreading of filamentous tau pathology in ALZ17 mice injected with brainstem extracts from mice transgenic for human P301S tau. Gallyas-Braak silver staining of brain regions (subiculum, optic tract and thalamus, medial lemniscus) at a distance from the injection sites 15 months postinjection. The sections were counterstained with hematoxylin. Scale bars, $50 \mu \mathrm{m}$. 
ber of neurons of the injected LC was significantly reduced over time, whereas no neurodegeneration was apparent in the contralateral LC, which seemed to be able to clear tau pathology. Induced tau pathology also spread along LC axons to regions with afferent and efferent connections. Surprisingly, although the injection of PFFs into the hippocampus induced tauopathy in the LC, their injection into the LC did not lead to hyperphosphorylation or aggregation of tau in the hippocampus. The EC (which receives efferent projections from the LC) also failed to develop tau pathology.

When presymptomatic P301S tau mice were injected with brain extracts from symptomatic animals (Ahmed et al. 2014), induced neurofibrillary pathology was first detected 2 weeks after unilateral injection and increased in a stereotypic and time-dependent manner. Contralateral and caudorostral propagation of tau pathology was evident in nuclei with strong efferent and afferent connections to the injection sites, revealing that the anatomical spread was dependent on connectivity not proximity. These findings were recently confirmed with the injection of PFFs in various brain regions of mice transgenic for P301S tau (line PS19) (Stancu et al. 2015). Inoculation of PFFs into the frontal cortex and hippocampus generated tau hyperphosphorylation and sarkosyl-insoluble tau aggregates. When the injection was placed in the EC, induced tauopathy spread to the connected hippocampus, amygdala, subiculum, thalamus, frontal cortex, and the contralateral hemisphere. When tau seeds were applied to basal ganglia, tau aggregation propagated to the striatum, thalamus, brainstem, and motor cortex. Induced tau pathology altered synaptic plasticity and led to cognitive and motor deficits. This study revealed that the pattern of the prion-like spreading of induced tau pathology was dependent on the initial site of PFF application and confirmed that pathological tau propagation occurred via connectivity rather than proximity.

Virally mediated expression of tau has also been used to investigate the propagation of pathology. Transneuronal spreading of tau pathology into the dentate gyrus has been observed following adeno-associated virus-mediated expression of P301L tau in layer II of the entorhinal cortex (Siman et al. 2013; Asai et al. 2015; Wegmann et al. 2015). The expression of mouse tau was not necessary (Wegmann et al. 2015). The spreading of wild-type tau has also been observed when lentiviral vectors were used ( Dujardin et al. 2014). It remains to be seen what the function of the intercellular spread of wild-type tau may be and if it interacts in any way with the spread of mutant tau.

We have shown that the intraperitoneal injection of brain extracts from symptomatic P301S tau mice into presymptomatic mice promoted the formation of cerebral tau inclusions (Fig. 2A) (Clavaguera et al. 2014). This indicates that, like prions and assembled $A \beta$ and $\alpha$-synuclein (Prusiner 1998; Eisele et al. 2010; Sacino et al. 2014), aggregated tau can promote inclusion formation in the central nervous system of transgenic mice following its peripheral administration.

\section{TAU STRAINS}

We showed that neuronal and glial tau filaments formed in ALZ17 mice following the intracerebral injection of brain homogenates from humans with pathologically confirmed tauopathies (Clavaguera et al. 2013a). Inclusions formed after inoculation of brain homogenates from all cases of $\mathrm{AD}, \mathrm{TD}, \mathrm{PiD}, \mathrm{AGD}, \mathrm{PSP}$, and CBD (Fig. 2B). Specific lesions reminiscent of human disorders were observed subsequent to the injection of brain homogenates from patients with the $4 \mathrm{R}$ tauopathies AGD, PSP, and CBD. With the exception of PiD, where tau inclusions are made predominantly of $3 \mathrm{R}$ tau, the inclusions of the other tauopathies used are made of either 4R tau (AGD, PSP, and CBD) or a mixture of $3 R$ and $4 R$ tau (AD and TD). The intracerebral injection of PSP brain homogenates into ALZ17 mice resulted in the formation of silver-positive aggregates that resembled tufted astrocytes (the hallmark lesions of PSP) (Nishimura et al. 1992; Yamada et al. 1992); the injection of CBD homogenates gave rise to the formation of silver-positive structures reminiscent of astrocytic plaques found in CBD (Feany 
F. Clavaguera et al.

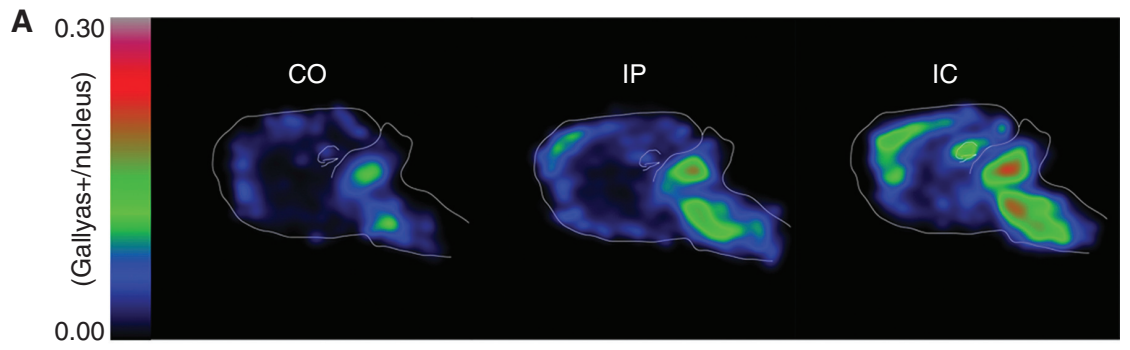

B

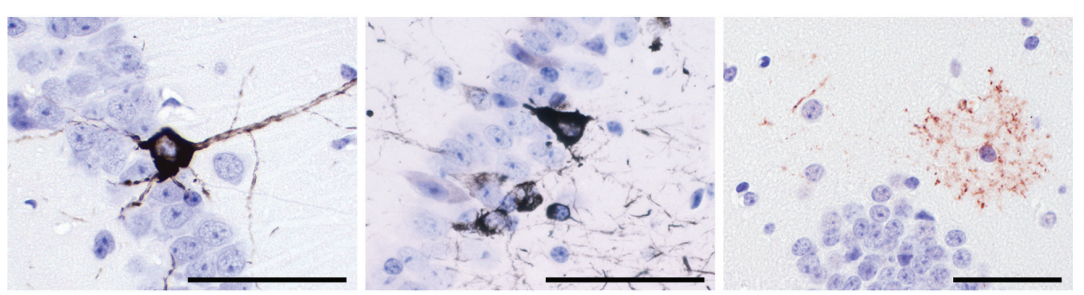

C

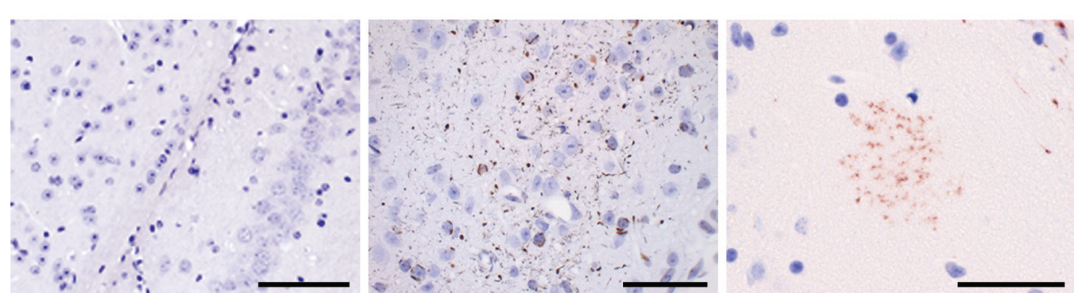

Figure 2. Prion-like properties of induced tau pathology. (A) 12-month-old heterozygous P301S tau mice 9 months after intraperitoneal (IP) or intracerebral (IC) injection with brainstem extracts from homozygous P301S tau mice. Gallyas-Braak silver-positive structures per cell nucleus ( $\mathrm{G} / \mathrm{N}$ ratios) in noninjected control mice (CO) and in IP- or IC-injected mice. Color maps representing average G/N ratios (CO, IP, and IC) of five sagittal brain sections per group. IP-injected mice had significantly higher $\mathrm{G} / \mathrm{N}$ ratios in the brainstem and neocortex than CO mice. The major difference between IP- and IC-injected animals was located in the hippocampus, one of the two intracerebral injection sites. (B) Filamentous tau lesions formed in the brains of ALZ17 mice after injection of human brain extracts. Gallyas-Braak silver staining revealed NFTs after the injection of $\mathrm{AD}$ brain homogenates (left) and argyrophilic grains after the inoculation of AGD brain homogenate (middle). AT100 immunolabeling detected a tau-positive tufted-like astrocyte consecutive to the injection of AGD brain homogenate (right). The sections were counterstained with hematoxylin. Scale bars, $50 \mu \mathrm{m}$. $(C)$ Induction of tau inclusions in nontransgenic C57Bl/6 mice 12 mo after intracerebral injection of brain homogenates from sporadic human tauopathies. Gallyas-Braak silver impregnation failed to detect tau filaments in noninjected mice (left) but revealed the presence of neuropil threads and coiled bodies in the dorsal thalamus following the injection of AD homogenate (middle). AT100 immunostaining of the hippocampal region showed the presence of a tufted-like astrocyte following the injection of AGD brain extract (right).

and Dickson 1995; Komori et al. 1998); and the injection of AGD homogenates resulted in the formation of silver-negative astrocytic tau inclusions like those observed in human AGD (Botez et al. 1999; Tolnay and Clavaguera 2004). In addition, with the exception of PiD, filamentous tau pathology propagated over time to some neighboring, as well as to connected distant, brain regions. Similar inclusions also formed after the intracerebral injection of brain homogenates from human tauopathies into nontransgenic mice (Fig. 2C). Moreover, serial propagation of induced filamentous tau pathology was observed when brain homogenates from ALZ17 mice that had received a bilateral injection of brain extracts from human P301S tau transgenic mice 18 months earlier were injected into 3-month-old ALZ17 mice. Twelve 
months after injection, Gallyas-Braak silver staining and AT100 immunostaining revealed the presence of neuronal and oligodendroglial tau inclusions at the injection sites. A second set of homogenates was prepared from the brains of nontransgenic mice that had been injected bilaterally with TD or AGD brain homogenates 18 months earlier. Twelve months after the intracerebral injection into ALZ17 mice, many NTs and tau aggregates in nerve cell bodies were present at the injection sites. These findings strongly suggest that different tau strains exist that are capable of inducing distinct tauopathies. Some of our findings were replicated when $\mathrm{AD}$ and $\mathrm{CBD}$ brain homogenates were injected into the right hippocampus and overlying cortex of PS19 mice (Boluda et al. 2015). CBD brain homogenates led to the formation of glial tau pathology in white-matter tracts and near the hippocampal injection site that increased with time and spread to the contralateral hemisphere. Coiled bodies were observed in the fimbria, alveus, subcortical white matter, and external capsule. Astrocytic plaques like those of human $\mathrm{CBD}$ were present in the stratum radiatum of the hippocampus in both hemispheres. A small amount of tau pathology was observed in some neuronal perikarya of CA1, CA3, and DG. AD brain homogenates led to the formation of tau inclusions in perikarya and processes of hippocampal neurons that propagated to rostral and caudal regions, as well as to the contralateral hippocampus. At later time points, tau pathology also developed in glial cells, but to a lesser extent when compared with injected CBD brain homogenates. Although no neurodegeneration was noticed following the injection of CBD brain extracts, the inoculation of $\mathrm{AD}$ extracts led to nerve cell loss in CA3. Detailed analysis of the induced tau pathology in PS19 mice after injection of AD and CBD extracts revealed strict immunological and histochemical similarities to the tau lesions of the human diseases. Similarly, conformationally distinct tau strains made of $4 \mathrm{R}$ tau repeats formed in HEK293 cells (Sanders et al. 2014). Inoculation of these strains into the hippocampus of young P301S tau mice induced pathologies that were stable through serial injection.

\section{MECHANISMS OF TAU PATHOLOGY INDUCTION AND PROPAGATION}

The assembly of soluble tau into filaments proceeds through energetically unfavorable nucleation (nucleated polymerization or nucleated conformational conversion) (Fig. 3) (Eisenberg and Jucker 2012). This leads to the formation of tau seeds, which grow by recruiting soluble monomeric tau into the inclusions. The nucleation phase of aggregation can be bypassed by the addition of exogenous seeds. In sporadic diseases, tau may aggregate into filaments in as little as a single cell. This may be the result of reduced turnover, potentially resulting from hyperphosphorylation or other modifications. Given enough time, these tau seeds may lead to filament formation in many cells distributed through the brain and give rise to disease symptoms. The studies discussed here do not tell us anything about the initial aggregation events, which we may never be able to predict. However, inhibition of the spreading of pathology could well lead to the prevention of clinical symptoms. It is therefore essential to understand the molecular mechanisms involved in the uptake, seeding, and release of tau inclusions.

The uptake of aggregated tau appears to rely on mechanisms of macropinocytosis and depends on the presence of cell-surface heparan sulphate proteoglycans (Kfoury et al. 2012; Holmes et al. 2013). Both monomeric and aggregated tau are taken up by cells, but only aggregated tau is able to seed the aggregation of soluble, monomeric tau (Falcon et al. 2015). We recently dissected the molecular characteristics of seed-competent tau species from the brains of symptomatic P301S tau mice (Jackson et al. 2016). We found that sucrose gradient fractions from brain lysates seeded tau aggregation in transfected cells only when large tau aggregates ( $>10$ mers) were present. There was no detectable seeding by fractions containing small, oligomeric ( $<6$ mers) tau aggregates, despite the presence of an apparently stable $400-\mathrm{kDa}$ form of tau on nondenaturing gels. Although more work needs to be performed, these findings suggest that P301S tau may aggregate by nucleated conformational conversion. Fractions contain- 
F. Clavaguera et al.
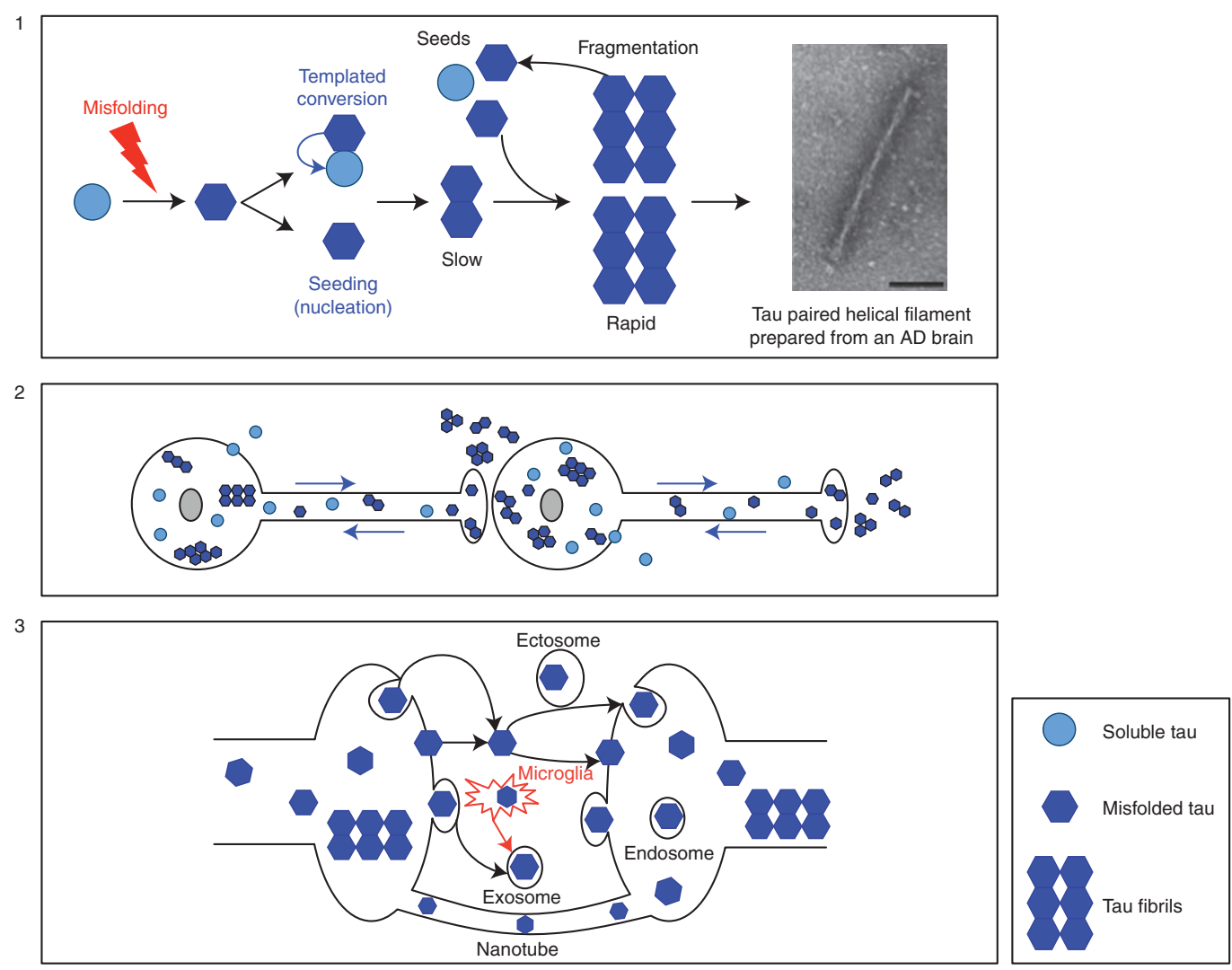

Figure 3. Possible mechanisms for tau-aggregate formation, intercellular transfer, and propagation. (1) Formation of tau filaments: For yet-unknown reasons, soluble wild-type tau (circle) can change its conformation ("misfolding") (hexagon). It is also able to adopt abnormal conformations after contact with abnormally folded tau (templated misfolding). Generation of oligomers by nucleation is a slow phenomenon. However, once a nucleus has formed, the growth of aggregates is rapid. Moreover, fragmentation of aggregates provides new seeds and accelerates further aggregation. Tau filaments are at the end point of aggregation and consist of amyloid fibrils rich in $\beta$-sheet structure, which can adopt different conformations that may be responsible for the heterogeneity of tauopathies. These conformations may correspond to distinct strains of aggregated tau. A paired helical filament from an AD brain is shown (Crowther and Goedert 2000). Scale bar, $100 \mathrm{~nm}$. (2) Interneuronal transfer: Tau may be released into the extracellular space in its soluble or misfolded form and/or be transported along the axon in both anterograde and retrograde directions. It may subsequently be taken up by neighboring cells, where tau filaments might induce aggregation of soluble tau in the cytoplasm. (3) Zoom on hypothetical mechanisms: Tau seeds circulate freely in the extracellular space or are contained in vesicles such as ectosomes or exosomes. They may penetrate neurons directly through the plasma membrane or by means of endocytosis. The transport of tau aggregates may also occur via nanotubes that connect the cytoplasm of adjoining cells.

ing large tau aggregates induced the formation and spreading of filamentous tau in presymptomatic mice transgenic for P301S tau, whereas fractions containing tau monomers and small aggregates were inactive. By immunoelectron microscopy, seed-competent sucrose gradient fractions contained aggregated tau species ranging from ring-like structures to small filaments.
These findings show that short fibrils are the major seed-competent tau species in the TgP301S tau mouse model. It has been said that it is essential to compare the effects of different assemblies at identical particle concentrations-because cells interact with particles not with the monomeric proteins that make up the particles (Melki 2015). Because oligomeric spe- 
cies are made of 10 to 50 tau monomers and 100 - to 200-nm fibrils of thousands of monomers, it follows that at equal concentrations of monomer, many more oligomers than filaments were injected (Jackson et al. 2016). It will be important to determine if similar species of aggregated tau underlie seeding, spreading, and neurodegeneration in $\mathrm{AD}$ and other human tauopathies. These findings are in contrast to a study reporting that a tau trimer was capable of seeding intracellular tau aggregation (Mirbaha et al. 2015).

Less is known about the mechanisms that underlie the release of aggregated tau. It is unknown what percentage of released tau aggregates is free and what is membrane-enclosed. This is important because immunotherapies will require access to tau aggregates. A recent study has reported that following viral expression of P301L tau, microglia internalize tau aggregates, which can be delivered to neurons via microglia-derived exosomes (Fig. 3C) (Asai et al. 2015). These findings suggest that tau propagation can occur through both transneuronal and nontransneuronal mechanisms. It will be important to dissect these contributions quantitatively. Although it is known that tau aggregates can spread between connected neurons, it is not known if their release is through synaptic mechanisms.

\section{CONCLUSION}

The findings summarized here point to similar intraorganismal pathological mechanisms in prion diseases and tauopathies. They favor the intercellular propagation of tau aggregates and support the existence of distinct tau strains that may explain the heterogeneity of human tauopathies. Misfolded tau seeds released into the extracellular space or transported via the axon can be taken up and therefore move from cell to cell; interfering with this process could stop disease progression (Grüninger 2015; Pedersen and Sigurdsson 2015; see also Holmes and Diamond 2017). There is substantial in vivo evidence for intracerebral cell-to-cell transmission of tau aggregates; however, it is essential to know if this is also related to neurodegeneration. With the exception of one study demonstrating the loss of CA1 neurons after the intracerebral injection of large amounts of synthetic tau fibrils into young P301L transgenic mice (Peeraer et al. 2015), nerve cell loss has not been observed following the injection of tau fibrils. It is possible that the propagation of tau inclusions, although necessary, is not sufficient for neurodegeneration. The tau species responsible for neurotoxicity may thus differ from those required for propagation (Clavaguera et al. 2009; Ahmed et al. 2014). Toxicity may require the generation of tau species by secondary nucleation (Cohen et al. 2013) after initial tau spreading and propagation; it may also depend on longer incubation times and/or larger amounts of seedcompetent tau. A better knowledge of the mechanisms that cause tau strain-specific onset and propagation via cell-to-cell transmission in the various tauopathies will pave the way to future diagnostic (Zhang et al. 2012; Maruyama et al. 2013; Okamura et al. 2014) and therapeutic (Grüninger 2015) options.

\section{ACKNOWLEDGMENTS}

We thank the following for financial support: UK Medical Research Council (U105184291), the Association pour la Recherche sur Alzheimer, and the Swiss National Science Foundation (31003A_152846).

\section{REFERENCES}

${ }^{*}$ Reference is also in this collection.

Ahmed Z, Cooper J, Murray TK, Garn K, McNaughton E, Clarke H, Parhizkar S, Ward MA, Cavallini A, Jackson S, et al. 2014. A novel in vivo model of tau propagation with rapid and progressive neurofibrillary tangle pathology: The pattern of spread is determined by connectivity, not proximity. Acta Neuropathol 127: 667-683.

Allen B, Ingram E, Takao M, Smith MJ, Jakes R, Virdee K, Yoshida H, Holzer M, Craxton M, Emson PC, et al. 2002. Abundant tau filaments and nonapoptotic neurodegeneration in transgenic mice expressing human P301S tau protein. J Neurosci 22: 9340-9351.

Asai H, Ikezu S, Tsunoda S, Medalla M, Luebke J, Haydar T, Wolozin B, Butovsky O, Kugler S, Ikezu T. 2015. Depletion of microglia and inhibition of exosome synthesis halt tau propagation. Nat Neurosci 18: 1584-1593.

Boluda S, Iba M, Zhang B, Raible KM, Lee VMY, Trojanowski JQ. 2015. Differential induction and spread of tau 
F. Clavaguera et al.

pathology in young PS19 tau transgenic mice following intracerebral injections of pathological tau from Alzheimer's disease or corticobasal degeneration brains. Acta Neuropathol 129: 221-237.

Botez G, Probst A, Ipsen S, Tolnay M. 1999. Astrocytes expressing hyperphosphorylated tau protein without glia fibrillary tangles in argyrophilic grain disease. Acta Neuropathol 98: $251-256$.

Braak H, Braak E. 1991. Neuropathological stageing of Alzheimer-related changes. Acta Neuropathol 82: 239-259.

Braak H, Del Tredici K. 2011. The pathological process underlying Alzheimer's disease in individuals under thirty. Acta Neuropathol 121: 171-181.

Braak H, Alafuzoff I, Arzberger T, Kretzschmar H, Del Tredici K. 2006. Staging of Alzheimer disease-associated neurofibrillary pathology using paraffin sections and immunocytochemistry. Acta Neuropathol 112: 389-404.

Clavaguera F, Bolmont T, Crowther RA, Abramowski D, Frank S, Probst A, Fraser G, Stalder AK, Beibel M, Staufenbiel M, et al. 2009. Transmission and spreading of tauopathy in transgenic mouse brain. Nat Cell Biol 11: 909-913.

Clavaguera F, Akatsu H, Fraser G, Crowther RA, Frank S, Hench J, Probst A, Winkler DT, Reichwald J, Staufenbiel M, et al. 2013a. Brain homogenates from human tauopathies induce tau inclusions in mouse brain. Proc Natl Acad Sci 110: 9535-9540.

Clavaguera F, Lavenir I, Falcon B, Frank S, Goedert M, Tolnay M. 2013b. "Prion-like" templated misfolding in tauopathies. Brain Pathol 23: 342-349.

Clavaguera F, Hench J, Lavenir I, Schweighauser G, Frank S Goedert M, Tolnay M. 2014. Peripheral administration of tau aggregates triggers intracerebral tauopathy in transgenic mice. Acta Neuropathol 127: 299-301.

Clavaguera F, Hench J, Goedert M, Tolnay M. 2015. Prionlike transmission and spreading of tau pathology. Neuropathol Appl Neurobiol 41: 47-58.

Cohen SIA, Linse S, Luheshi LM, Hellstrand E, White DA, Rajah L, Otzen DE, Vendruscolo M, Dobson CM, Knowles TPJ. 2013. Proliferation of amyloid- $\beta 42$ aggregates occurs through a secondary nucleation mechanism. Proc Natl Acad Sci 110: 9758-9763.

Crowther RA, Goedert M. 2000. Abnormal tau-containing filaments in neurodegenerative diseases. J Struct Biol 130: 271-279.

de Calignon A, Polydoro M, Suarez-Calvet M, William C, Adamowicz DH, Kopeikina KJ, Pitstick R, Sahara N, Ashe KH, Carlson GA, et al. 2012. Propagation of tau pathology in a model of early Alzheimer's disease. Neuron 73: 685-697.

Delacourte A, Robitaille Y, Sergeant N, Bué L, Hof PR, Wattez A, Laroche-Cholette A, Mathieu J, Chagnon P, Gauvreau D. 1996. Specific pathological Tau protein variants characterize Pick's disease. J Neuropathol Exp Neurol 55: $159-168$.

Dujardin S, Lecolle K, Caillierez R, Begard S, Zommer N, Lachaud C, Carrier S, Dufour N, Auregan G, Winderickx J, et al. 2014. Neuron-to-neuron wild-type Tau protein transfer through a trans-synaptic mechanism: Relevance to sporadic tauopathies. Acta Neuropathol Commun 2: 14.
Eisele YS, Obermüller U, Heilbronner G, Baumann F, Kaeser SA, Wolburg H, Walker LC, Staufenbiel M, Heikenwalder M, Jucker M. 2010. Peripherally applied A $\beta$-containing inoculates induce cerebral $\beta$-amyloidosis. Science 330: 980-982.

Eisenberg D, Jucker M. 2012. The amyloid state of proteins in human diseases. Cell 148: 1188-1203.

Falcon B, Cavallini A, Angers R, Glover S, Murray TK, Barnham L, Jackson S, O'Neill MJ, Isaacs AM, Hutton ML, et al. 2015. Conformation determines the seeding potencies of native and recombinant Tau aggregates. J Biol Chem 290: $1049-1065$.

Feany MB, Dickson DW. 1995. Widespread cytoskeletal pathology characterizes corticobasal degeneration. Am Pathol 146: 1388-1396.

Flament S, Delacourte A, Verny M, Hauw JJ, Javoy-Agid F. 1991. Abnormal Tau proteins in progressive supranuclear palsy. Similarities and differences with the neurofibrillary degeneration of the Alzheimer type. Acta Neuropathol 81: 591-596.

Ghetti B, Wszolek ZW, Boeve BF, Spina S, Goedert M. 2011. Frontotemporal dementia and parkinsonism linked to chromosome 17. In Neurodegeneration: The molecular pathology of dementia and movement disorders, 2nd ed. (ed. Dickson DW, Weller RO), pp. 110-134. WileyBlackwell, Oxford.

Goedert M. 2015. Alzheimer's and Parkinson's diseases: The prion concept in relation to assembled $\mathrm{A} \beta$, tau, and $\alpha$ synuclein. Science 349: 1255555.

Goedert M, Jakes R. 1990. Expression of separate isoforms of human tau protein: Correlation with the tau pattern in brain and effects on tubulin polymerization. EMBO J 9: 4225-4230.

Goedert M, Spillantini MG, Jakes R, Rutherford D, Crowther RA. 1989. Multiple isoforms of human microtubule-associated protein tau: Sequences and localization in neurofibrillary tangles of Alzheimer's disease. Neuron 3: $519-526$.

Goedert M, Spillantini MG, Cairns NJ, Crowther RA. 1992. Tau proteins of Alzheimer paired helical filaments: Abnormal phosphorylation of all six brain isoforms. Neuron 8: $159-168$.

Goedert M, Clavaguera F, Tolnay M. 2010. The propagation of prion-like protein inclusions in neurodegenerative diseases. Trends Neurosci 33: 317-325.

Grüninger F. 2015. Drug development for tauopathies. Neuropathol Appl Neurobiol 41: 81-96.

* Holmes BB, Diamond MI. 2017. Cellular models for the study of prions. Cold Spring Harb Perspect Med 7: a024026.

Holmes BB, DeVos SL, Kfoury N, Li M, Jacks R, Yanamandra K, Ouidja MO, Brodsky FM, Marasa J, Bagchi DP, et al. 2013. Heparan sulfate proteoglycans mediate internalization and propagation of specific proteopathic seeds. Proc Natl Acad Sci 110: E3138-E3147.

Hutton M, Lendon CL, Rizzu P, Baker M, Froelich S, Houlden $\mathrm{H}$, Pickering-Brown $\mathrm{S}$, Chakraverty $\mathrm{S}$, Isaacs $\mathrm{A}$, Grover A, et al. 1998. Association of missense and 5'splice site mutations in tau with the inherited dementia FTDP-17. Nature 393: 702-705. 
Prion-Like Behavior of Assembled Tau in Transgenic Mice

Iba M, Guo JL, McBride JD, Zhang B, Trojanowski JQ, Lee VMY. 2013. Synthetic tau fibrils mediate transmission of neurofibrillary tangles in a transgenic mouse model of Alzheimer's-like tauopathy. J Neurosci 33: 1024-1037.

Iba M, McBride JD, Guo JL, Zhang B, Trojanowski JQ, Lee VMY. 2015. Tau pathology spread in PS19 tau transgenic mice following locus coeruleus (LC) injections of synthetic tau fibrils is determined by the LC's afferent and efferent connections. Acta Neuropathol 130: 249-362.

Jackson SI, Kerridge K, Cooper J, Cavallini A, Falcon B, Cella CV, Land IA, Szekeres PG, Murray TK, Ahmed Z, et al. 2016. Short fibrils constitute the major species of seedcompetent tau in the brains of mice transgenic for human P301S tau. J Neurosci 36: 762-772.

Kfoury N, Holmes BB, Jiang H, Holtzman DM, Diamond M. 2012. Transcellular propagation of tau aggregation by fibrillar species. J Biol Chem 287: 19940-19451.

Komori T, Arai N, Oda M, Nakayama H, Mori H, Yagishita S, Takahashi T, Amano N, Murayama S, Murakami S, et al. 1998. Astrocytic plaques and tufts of abnormal fibers do not coexist in corticobasal degeneration and progressive supranuclear palsy. Acta Neuropathol 96: 401-408.

Kovacs GG. 2015. Neuropathology of tauopathies: Principles and practice. Neuropathol Appl Neurobiol 41: 3-23.

Ksiezak-Reding H, Morgan K, Mattiace LA, Davies P, Liu WK, Yen SH, Weidenheim K, Dickson DW. 1994. Ultrastructure and biochemical composition of paired helical filaments in corticobasal degeneration. Am J Pathol 145: 1496-1508.

Lasagna-Reeves CA, Castillo-Carranza DL, Sengupta U, Guerrero-Munoz MJ, Kiritoshi T, Neugebauer V, Jackson GR, Kayed R. 2012. Alzheimer brain-derived tau oligomers propagate pathology from endogenous tau. Sci Rep 2: 700 .

Liu L, Drouet V, Wu JW, Witter MP, Small SA, Clelland C, Duff K. 2012. Trans-synaptic spread of tau pathology in vivo. PLoS ONE 7: e31302.

Maruyama M, Shimada H, Suhara T, Shinotoh H, Ji B, Maeda J, Zhang MR, Trojanowski JQ, Lee VMY, Ono $\mathrm{M}$, et al. 2013. Imaging of tau pathology in a tauopathy mouse model and in Alzheimer patients compared to normal controls. Neuron 79: 1094-1108.

McKee AC, Stern RA, Nowinski CJ, Stein TD, Alvarez VE, Daneshvar DH, Lee HS, Wojtowicz SM, Hall G, Baugh $\mathrm{CM}$, et al. 2013. The spectrum of disease in chronic traumatic encephalopathy. Brain 136: 43-64.

Melki R. 2015. Role of different $\alpha$-synuclein strains in synucleinopathies, similarities with other neurodegenerative diseases. J Parkinsons Dis 5: 217-227.

Mirbaha H, Holmes BB, Sanders DW, Bieschke J, Diamond MI. 2015. Tau trimers are the minimal propagation unit spontaneously internalized to seed intracellular aggregation. J Biol Chem 290: 14893-14903.

Nishimura M, Namba Y, Ikeda K, Oda M. 1992. Glial fibrillary tangles with straight tubules in the brains of patients with progressive supranuclear palsy. Neurosci Lett 143: 35-38.

Noda K, Sasaki K, Fujimi K, Wakisaka Y, Tanizaki Y, Wakugawa Y, Kiyohara Y, Iida M, Aizawa H, Iwaki T. 2006. Quantitative analysis of neurofibrillary pathology in a general population to reappraise neuropathological criteria for senile dementia of the neurofibrillary tangle type (tangle-only dementia): The Hisayama study. Neuropathology 26: 508-518.

Okamura N, Furumoto S, Fodero-Tavoletti MT, Mulligan RS, Harada R, Yates P, Pejoska S, Kudo Y, Masters CL, Yanai K, et al. 2014. Non-invasive assessment of Alzheimer's disease neurofibrillary pathology using $18 \mathrm{~F}-$ THK5105 PET. Brain 137: 1762-1771.

Pedersen JT, Sigurdsson EM. 2015. Tau immunotherapy for Alzheimer's disease. Trends Mol Med 21: 394402.

Peeraer E, Bottelbergs A, Van Kolen K, Stancu IC, Vasconcelos B, Mahieu M, Duytschaever H, Ver Donck L, Torremans A, Sluydts E, et al. 2015. Intracerebral injection of preformed synthetic tau fibrils initiates widespread tauopathy and neuronal loss in the brains of tau transgenic mice. Neurobiol Dis 73: 83-95.

Poorkaj P, Bird TD, Wijsman E, Nemens E, Garruto RM, Anderson L, Andreadis A, Wiederholt WC, Raskind M, Schellenberg GD. 1998. Tau is a candidate gene for chromosome 17 frontotemporal dementia. Ann Neurol 43: 815-825.

Probst A, Langui D, Lautenschlager C, Ulrich J, Brion JP, Anderton BH. 1988. Progressive supranuclear palsy: Extensive neuropil threads in addition to neurofibrillary tangles. Very similar antigenicity of subcortical neuronal pathology in progressive supranuclear palsy and Alzheimer's disease. Acta Neuropathol 77: 61-68.

Probst A, Tolnay M, Langui D, Goedert M, Spillantini MG. 1996. Pick's disease: Hyperphosphorylated tau protein segregates to the somatoaxonal compartment. Acta Neuropathol 92: 588-596.

Probst A, Götz J, Wiederhold KH, Tolnay M, Mistl C, Jaton AL, Hong M, Ishihara T, Lee VMY, Trojanowski JQ, et al. 2000. Axonopathy and amyotrophy in mice transgenic for human four-repeat tau protein. Acta Neuropathol 99: 469-481.

Prusiner SB. 1998. Prions. Proc Natl Acad Sci 95: 13363 13383.

Sacino AN, Brooks M, Thomas MA, McKinney AB, Lee S, Regenhardt RW, McGarvey NH, Ayers JI, Notterpek L, Borchelt DR, et al. 2014. Intramuscular injection of $\alpha$-synuclein induces CNS $\alpha$-synuclein pathology and a rapid-onset motor phenotype in transgenic mice. Proc Natl Acad Sci 111: 10732-10737.

Saito Y, Ruberu NN, Sawabe M, Arai T, Tanaka N, Kakuta Y, Yamanouchi H, Murayama S. 2004. Staging of argyrophilic grains: An age-associated tauopathy. J Neuropathol Exp Neurol 63: 911-918.

Sanders DW, Kaufman SK, DeVos SL, Sharma AM, Mirbaha H, Li A, Barker SJ, Foley AC, Thorpe JR, Serpell LC, et al. 2014. Distinct tau prion strains propagate in cells and mice and define different tauopathies. Neuron 82: 1271-1288.

Schmidt ML, Zhukareva V, Newell KL, Lee VM, Trojanowski JQ. 2001. Tau isoform profile and phosphorylation state in dementia pugilistica recapitulate Alzheimer's disease. Acta Neuropathol 101: 518-524.

Siman R, Lin YG, Malthankar-Phatak G, Dong Y. 2013. A rapid gene delivery-based mouse model for early-stage Alzheimer disease-type tauopathy. J Neuropathol Exp Neurol 72: 1062-1071. 
F. Clavaguera et al.

Spillantini MG, Goedert M. 2013. Tau pathology and neurodegeneration. Lancet Neurol 12: 609-622.

Spillantini MG, Murrell JR, Goedert M, Farlow MR, Klug A Ghetti B. 1998. Mutation in the tau gene in familial multiple system tauopathy with presenile dementia. Proc Natl Acad Sci 95: 7737-7741.

Stancu IC, Vasconcelos B, Ris L, Wang P, Villers A, Peeraer E, Buist A, Terwel D, Baatsen P, Oyelami T, et al. 2015. Templated misfolding of Tau by prion-like seeding along neuronal connections impairs neuronal network function and associated behavioral outcomes in Tau transgenic mice. Acta Neuropathol 129: 875-894.

Togo T, Sahara N, Yen SH, Cookson N, Ishizawa T, Hutton M, de Silva R, Lees A, Dickson DW. 2002. Argyrophilic grain disease is a sporadic 4-repeat tauopathy. J Neuropathol Exp Neurol 61: 547-556.

Tolnay M, Clavaguera F. 2004. Argyrophilic grain disease: A late-onset dementia with distinctive features among tauopathies. Neuropathology 24: 269-283.
Tolnay M, Probst A. 2002. Frontotemporal lobar degeneration-Tau as a pied piper? Neurogenetics 4: 63-75.

Tolnay M, Sergeant N, Ghestem A, Chalbot S, De Vos RA, Jansen Steur EN, Probst A, Delacourte A. 2002. Argyrophilic grain disease and Alzheimer's disease are distinguished by their different distribution of tau protein isoforms. Acta Neuropathol 104: 425-434.

Wegmann S, Maury EA, Kirk MJ, Saqran L, Roe A, DeVos SL, Nicholls S, Fan Z, Takeda S, Cagsal-Getkin O, et al. 2015. Removing endogenous tau does not prevent tau propagation yet reduces its neurotoxicity. EMBO J 34: 3028-3041.

Yamada T, McGeer PL, McGeer EG. 1992. Appearance of paired nucleated, Tau-positive glia in patients with progressive supranuclear palsy brain tissue. Neurosci Lett 135: 99-102.

Zhang W, Arteaga J, Cashion DK, Chen G, Gangadharmath U, Gomez LF, Kasi D, Lam C, Liang Q, Liu C, et al. 2012. A highly selective and specific PET tracer for imaging of tau pathologies. J Alzheimers Dis 31: 601-612. 


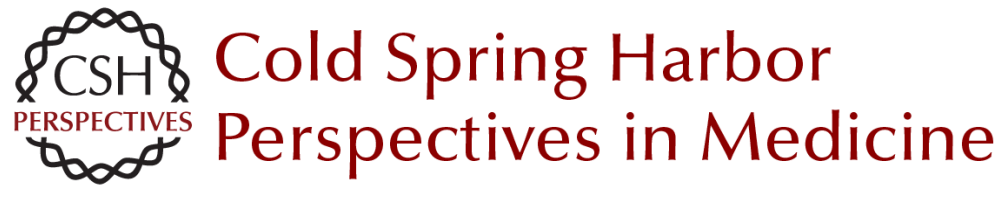

\section{The Prion-Like Behavior of Assembled Tau in Transgenic Mice}

Florence Clavaguera, Markus Tolnay and Michel Goedert

Cold Spring Harb Perspect Med 2017; doi: 10.1101/cshperspect.a024372 originally published online December 9, 2016

\section{Subject Collection Prion Diseases}

TDP-43 Prions

Takashi Nonaka and Masato Hasegawa

$\alpha$-Synuclein: Multiple System Atrophy Prions Amanda L. Woerman, Joel C. Watts, Atsushi Aoyagi, et al.

Genetics of Synucleinopathies Robert L. Nussbaum

$\beta$-Amyloid Prions and the Pathobiology of

Alzheimer's Disease Joel C. Watts and Stanley B. Prusiner

Disease Mechanisms of C9ORF72 Repeat Expansions Tania F. Gendron and Leonard Petrucelli

Chronic Traumatic Encephalopathy: Is Latency in Symptom Onset Explained by Tau Propagation? Joshua Kriegel, Zachary Papadopoulos and Ann C. McKee

Noncerebral Amyloidoses: Aspects on Seeding, Cross-Seeding, and Transmission Gunilla T. Westermark, Marcus Fändrich, Katarzyna Lundmark, et al.

Structural and Chemical Biology of Presenilin Complexes

Douglas S. Johnson, Yue-Ming Li, Martin

Pettersson, et al.
Cell Biology and Pathophysiology of $\alpha$-Synuclein Jacqueline Burré, Manu Sharma and Thomas C. Südhof

Molecular Mechanisms of Chronic Wasting

Disease Prion Propagation Julie A. Moreno and Glenn C. Telling

Genetics of Amyotrophic Lateral Sclerosis Mehdi Ghasemi and Robert H. Brown, Jr.

The Genetics of C9orf72 Expansions Ilse Gijselinck, Marc Cruts and Christine Van Broeckhoven

Prion-Like Characteristics of Polyglutamine-Containing Proteins Margaret M.P. Pearce and Ron R. Kopito

Therapeutic Strategies for Restoring Tau Homeostasis

Zapporah T. Young, Sue Ann Mok and Jason E. Gestwicki

Fused in Sarcoma Neuropathology in Neurodegenerative Disease Ian R.A. Mackenzie and Manuela Neumann

Experimental Models of Inherited PrP Prion Diseases Joel C. Watts and Stanley B. Prusiner

For additional articles in this collection, see http://perspectivesinmedicine.cshlp.org/cgi/collection/ 